\title{
Differential rotation on active late-type stars observed with Corot
}

\author{
P. Gondoin ${ }^{1}$, M. Fridlund ${ }^{1}$, D. Gandolfi ${ }^{2}$ and E. Güenter ${ }^{2}$ \\ ${ }^{1}$ European Space Agency, ESTEC - Postbus 299, 2200 AG Noordwijk, The Netherlands \\ ${ }^{2}$ Thüringer Landessternwarte Tautenburg, Sternwarte 5, 07778 Tautenburg, Germany
}

\begin{abstract}
One major topic in studying stellar activity is to explain how phenomena seen on the Sun and stars, and specially magnetic phenomena, depend on stellar properties such as rotation and age. Differential rotation is an important physical process in theories of stellar magnetic field generation. The solar surface differential rotation was initially discovered via the simple method of tracking the rotation rates of individual starspots at different latitudes. Adopting a similar principle, high accuracy light curves of active stars observed with the CoRoT satellite are analyzed using a model based on the rotational modulation of the visibility of active regions.
\end{abstract}

Keywords. stars: activity, stars: late-type, stars: rotation, stars: spots

\section{Introduction}

Differential rotation measurements have been performed on $\mathrm{F}$ and early $\mathrm{G}$ dwarfs (e.g. Reiners \& Schmitt 2003; Reiners 2006) by studying the rotational broadening of spectral lines with a Fourier transform method. Differential rotation has also been measured using Doppler imaging techniques on a few young rapidly rotating $\mathrm{G}$ and $\mathrm{K}$ stars (e.g. Donati et al. 2000; Petit et al. 2002; Collier-Cameron \& Donati 2002; Marsden et al. 2004; Barnes et al. 2005).

One magnetic-field diagnostic for cool stars is the presence of starspots. Starspots cooler than the unperturbed photosphere can account for the rotation modulated attenuation of stellar fluxes in broad optical pass-bands. The photometric periods of active stars have been measured by studying the rotation modulation of long sequences of broadband photometric measurements (e.g. Hall 1991; Henry et al. 1995; Messina \& Guinan 2003). We have analyzed high accuracy light curves of active F, G and K stars, that were obtained with the CoRoT satellite during its initial run.

\section{Lightcurves analyses}

The CoRoT satellite (Baglin et al. 2006; Auvergne et al. 2009) simultaneously registers the visible lightcurves of a large number of stars for durations of several months with the aim to detect extrasolar planets by the transit method. CoRoT observations started with a pointing direction close to the anticenter of the Galaxy. This initial run lasted from February $6^{\text {th }}$ to April $2^{\text {nd }} 2007$ and supplied high accuracy lightcurves. These time series are nearly continuous over 57 days with only a small number of gaps that mainly result from the crossing of the South-Atlantic Anomaly. A fraction of these light curves have a periodic modulation indicative of starspots.

The rotation modulation of stellar photometric lightcurves by stellar active regions can be modelled using two numerical approaches: the surface integration methods and the analytical method (Ribarik et al. 2003). The former assigns a temperature to each pixel of the spherical integration net and then varies each value until an optimal fit to 
the data is achieved. In this study, we use the analytical approach described by Lanza et al. (2006) and based on a model used to fit the time variations of the solar bolometric and spectral irradiance.

The relative flux variations of active stars are fitted with a two or three spots model. Best fit models to the lightcurves are obtained by minimizing the sum of squared residuals. The fixed parameters in the simulations included stellar parameters determined spectroscopically (Gandolfi et al. 2009) and active region parameters. The surfaces, longitudes, latitudes and rotation periods of the active regions are left as free parameters. The analyses of the lightcurves are performed as a grid search using a two or three spots model and iterating on 8 or 12 variable parameters. Series of fitting processes varying the inclination of the star rotation axis are conducted in order to identify the inclination angle that minimizes the overall $\chi^{2}$ and the fitting parameters for which the difference in $\chi^{2}$ becomes significant at a more than $80 \%$ confidence levels.

\section{Preliminary results}

Preliminary analysis were conducted on a sample of active dwarfs that were arbitrarily selected. One first result of this analysis is that all high accuracy lightcurves with evidence for rotation modulation cannot be parameterized with a simple two or three spots model. This may be due to complex topologies, time evolution or latitude migration of the active centers during the observing run. Among an initial sample of six dwarfs, the lightcurve of only one star could be fitted with a two spots model on the entire 57 days observation duration (see Fig. 1 top). The lightcurves of two other sample stars (see Fig. 1 middle and bottom) could be fitted on a large fraction (about 45 days) of the observing run.

Nevertheless, within the frame of the analysis method, differential rotation has been detected at a significant confidence level on these three stars. The differential rotations $\Delta P$ between the high and low latitude spots are $0.03,0.42$ and 0.5 days for average rotation periods of 3.05, 4.24 and 15.23 days, respectively. Although these first results may be underestimates of the latitudinal differential rotation shear, comparisons with measurements obtained with different analysis methods support the trend of increasing differential rotation rate $\Delta P / P$ with increasing rotation period.

\section{References}

Auvergne, M., Bodin, P., Boisnard, L. et al. 2009, A\&A, in press

Baglin, A., Auvergne, M., Boisnard, L., et al. 2006, 36th COSPAR Scientific Assembly, 36, 3749

Barnes, J. R., Collier Cameron, A., Donati, J.-F., et al. 2005, MNRAS, 357, L1

Collier Cameron, A. \& Donati, J.-F. 2002, MNRAS, 329, L23

Donati, J.-F., Mengel, M., Carter, B., et al. 2000, MNRAS, 316, 699

Gandolfi, D. et al. 2009, in preparation.

Hall, D. S. 1991, in Tuominen I., Moss D., Rüdiger G., (eds), Proc. IAU Colloq. 130, The Sun and Cool Stars, Springer-Verlag, p. 353

Henry, G. W., Eaton, J. A., Hamer, J., \& Hall, D. S., 1995, ApJS, 97, 513

Marsden, S. C., Waite, I. A., Carter, B. D., \& Donati, J.-F. 2004, AN, 325, 246

Lanza, A. F., Messina, S., Pagano, I., et al. 2006, AN, 327, 28

Petit, P., Donati, J.-F., Collier Cameron, A. 2002, MNRAS, 334, 374

Reiners, A. \& Schmitt, J. H. M. M. 2003, $A \& A$, 398, 647

Reiners, A. 2006, $A \& A$, 446, 267

Ribarik, G., Olah, K., \& Strassmeier, K. G. 2003, AN, 324, 202 

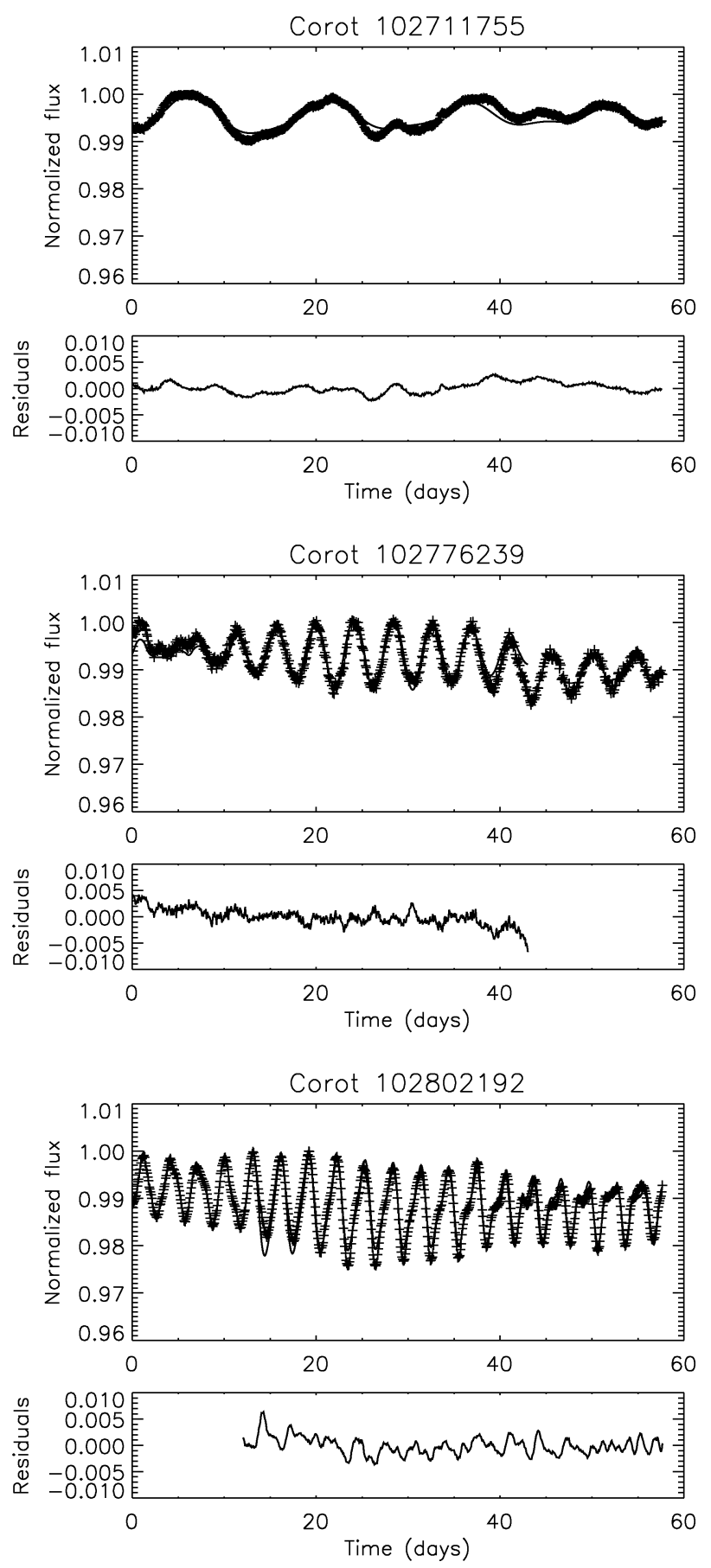

Figure 1. Best fit models and residuals to the light curve of a sample of active dwarfs observed with Corot. 